\title{
Thirty day results of 227 consecutive carotid stent procedures performed in carotid stenting clinical trials
}

\author{
D Heck
}

Forsyth Medical Center, Winston-Salem, North Carolina, USA

\section{Correspondence to} D Heck, Forsyth Medical Center, 3155 Maplewood Avenue, Winston-Salem, NC 27103, USA; dvheck@triad.rr.com

Received 16 August 2009 Accepted 18 August 2009 Published Online First 5 October 2009

\begin{abstract}
Background and purpose As evidence accumulates that carotid artery stenting (CAS) is a durable and effective procedure for stroke prevention, the utility of the procedure hinges on the perioperative risk. The perioperative risk of CAS procedures has historically been higher than carotid endarterectomy in most clinical trials. The perioperative risk of the series presented here is lower than any previously reported in the context of a clinical trial.
\end{abstract}

Methods Data were collected prospectively from 227 consecutive elective CAS procedures by a single neurointerventionalist performed as part of carotid stenting clinical trials as per the trial protocols, which included randomized trials of "average risk" patients and non-randomized trials of "high risk" patients. The primary outcome measures were 30 day stroke and death, and in most cases 30 day myocardial infarction (MI) also.

Results Follow-up was 100\%. There were no deaths. There was one technical failure. The overall 30 day stroke/death/Ml rate was 5/226 (2.2\%). There was one $\mathrm{Ml}$. The 30 day stroke and death rate for symptomatic patients was $2.5 \%$ and for asymptomatic patients $1.4 \%$. Two of the four strokes occurred in vascular territories separate from the target carotid artery.

Conclusions The perioperative CAS complications in this series were well below the $6 \%$ for symptomatic patients and $3 \%$ for asymptomatic patients currently recommended based on historic carotid endarterectomy data. Carotid stenting can be performed safely in the community, provided there are experienced interventionalists and proper patient selection.

Large randomized clinical trials have established carotid endarterectomy (CEA) as the standard treatment for symptomatic and asymptomatic severe carotid artery stenosis. ${ }^{1-4}$ Carotid artery stenting (CAS) has the potential advantage of being less invasive than CEA. One potential disadvantage of CAS is durability, but severe re-stenosis rates requiring retreatment have been low, of the order of $3 \%{ }^{5-7}$ The ability of carotid stenting to prevent stroke was also not initially known, but the stroke rates in symptomatic patients after the first 30 days until 1 year in the lead-in data from the Carotid Revascularization Endarterectomy versus Stenting Trial (CREST) trial have been similar to those reported in the North American Symptomatic Carotid Endarterectomy Trial (NASCET), ${ }^{8}$ and the stroke rates in randomized trials such as the StentSupported Percutaneous Angioplasty of the Carotid Artery versus Endarterectomy (SPACE) and Stenting and Angioplasty with Protection in Patients at High Risk for Endarterectomy
SAPPHIRE trials have been similar in the CEA and CAS groups after the first 30 days. ${ }^{9} 10$ Hence, with evidence that CAS is both a durable and effective procedure for the prevention of stroke, at least in the first 2-3 years, the utility of the procedure hinges on the perioperative risk.

Enthusiasm for CAS in patients at higher than average risk for CEA grew with publication of the SAPPHIRE trial using Precise/Angioguard (Cordis Endovascular, Bridgewater, New Jersey, USA), a randomized trial demonstrating a lower 30 day stroke, death and myocardial infarction (MI) rate for CAS compared with CEA in certain populations with higher than normal risk for CEA due to anatomic or cardiovascular risk factors. ${ }^{11}$ The 30 day stroke, death and MI rates for CEA and CAS were $4.4 \%$ and $9.9 \%$, respectively, the difference being due to non-Q wave MI. Most patients (70\%) were asymptomatic. This, in large part, led to CMS (Centers for Medicare Services) approval in 2005 of CAS for severe symptomatic stenosis in 'high risk' patients. Since then, published non-randomized premarket approval and post-market surveillance CAS trials concerning the 'high risk' population have had variable results, but generally worse than SAPPHIRE. ${ }^{12-16}$ The BEACH trial (Boston Scientific EPI: A Carotid Stenting Trial for High Risk Surgical Patients) investigated the use of the carotid Wallstent and filter-wire EX/EZ (Boston Scientific, Natick, Massachusetts, USA) in 'high risk' patients, $75 \%$ of whom were asymptomatic. The 30 day stroke, death and MI rate was $5.8 \%$ overall, $5 \%$ for asymptomatic and $7.9 \%$ for symptomatic patients. ${ }^{12}$ These events were mostly due to stroke and death, as the MI rate was less than $1 \%$. The complication rates were higher than the randomized CEA trials for asymptomatic patients, ${ }^{34}$ and slightly higher for symptomatic patients. ${ }^{1-2}{ }^{16-17}$ Results in the Acculink for Revascularization of Carotids in High Risk Patients (ARCHeR) trial evaluating protected CAS in high risk patients using Acculink/Accunet (Abbott Vascular, Santa Clara, California, USA) were worse, with 30 day major adverse event rates of $6.8 \%$ for asymptomatic and $13 \%$ for symptomatic patients. $^{13}$ The Carotid Acculink/Accunet PostApproval Trial to Uncover Unanticipated or Rare Events (CAPTURE) trial, also using Acculink/ Accunet, was designed to assess the safety of CAS in 'high risk' patients performed by physicians of varying specialty and expertise in the community. While the authors concluded that "transfer of this new therapy to the community practice setting via carotid stent training programs is effective", it is difficult to reconcile this statement with the results of CAPTURE. The CAPTURE population was $86 \%$ asymptomatic, and those patients had a 30 day 
stroke, death and MI rate of $5.4 \%$. Symptomatic patients fared much worse, with a 30 day stroke, death and MI rate of $12 \%{ }^{14}$ The fact that the results are similar to ARCHeR cannot really be that encouraging given the high complication rates in ARCHeR, especially for symptomatic patients. Operators were categorized as level 1, 2 or 3, in decreasing order of experience. The level 2 and 3 operators had 30 day fatality rates of $1.8 \%$ and $2.3 \%$, respectively, and a major stroke and death rate of $2.9 \%$ and $3.4 \%$, respectively. While the authors of CAPTURE reported no statistically significant difference in outcomes between the three groups, the trend in every important outcome measure except MI was towards higher complications in the lesser experienced operators. The Carotid Revascularization with EV3 Technology Evolution (CREATE) trial utilizing the Spider embolic protection device and the Protege stent (EV3, Plymouth, Minnesota, USA) had similarly poor results in the symptomatic group, with a 30 day stroke, death and MI rate of $16 \%$, but fared better in the asymptomatic group at $4 \% .{ }^{15}$ Technical failure occurred in $3 \%$ of patients. Considering that patients are generally not considered enrolled in these trials until the protection device is inserted, thus not accounting for the risk of angiography and guide catheter placement, the major adverse event rates if considered from the arterial puncture may actually be even higher.

Randomized trials comparing CAS and CEA in average risk symptomatic patients have had variable results. The SPACE trial failed to establish the non-inferiority of CAS compared with CEA although there was no significant difference in 30 day stroke and death between the techniques (6.3\% for CEA and $6.8 \%$ for CAS) ${ }^{16}$ A second randomized trial (EVA-3S) comparing CAS and CEA in symptomatic patients was stopped early due to almost threefold higher stroke and death rate of CAS $(9.6 \%$ compared with 3.4\%). ${ }^{17}$ EVA-3S has been criticized for the lack of experience of the CAS operators. The lead-in data from CREST, an average risk population for CEA, revealed a 30 day stroke and death event rate of $5.7 \%$ in symptomatic patients, similar to the risk of CEA in NASCET. The 30 day stroke and death rate in asymptomatic patients was $3.8 \%{ }^{18}$ The event rates were heavily influenced by higher rates in octogenarians. ${ }^{19}$

Given the well established 5 year ipsilateral stroke rate of just $11 \%$ for asymptomatic patients treated medically in both the Asymptomatic Carotid Atherosclerosis Study (ACAS) and the Asymptomatic Carotid Surgery Trial (ACST), ${ }^{3} 4$ 30-day major adverse event rates in asymptomatic patients routinely in excess of $3 \%$, as observed in all of the published pre-market approval and post-market surveillance trials, would nullify benefit in this patient group. Similarly, excessive 30 day major adverse event rates in symptomatic patients observed in ARCHeR, CAPTURE, CREATE and EVA-3S would neutralize any expected benefit of the procedure. These facts no doubt contribute to the current CMS position not to further extend coverage for carotid stenting.

The data presented here reflect a prospective, consecutive case series in a community practice where CAS has been adopted by the medical and neuroscience community with favorable results, and 30 day adverse event rates very favorable when compared with published trial data.

\section{METHODS}

The series constitutes 226 consecutive elective carotid interventions performed as part of a clinical trial by a single interventional neuroradiologist (DH) between March 2006 and June 2009. The patients reported here were enrolled in post-market approval trials (Emboshield and Xact Post Approval Carotid Stent Trial (EXACT), CAPTURE II, Carotid Stenting For High Surgical Risk Patients (CHOICE), SAPPHIRE-WW), pre-market approval trials (Protection During Carotid Stenting in High Risk Patients With the TriActiv ProGuard System (PROGUARD)) or randomized trials. There was one technical failure due to inability to cross the lesion. That patient had an elective CEA procedure and did not have a neurologic event. For the randomized trial patients, no patient randomized to CAS had an event prior to the procedure. For the post-market and pre-market approval trials, there were no patients who had a neurologic event during arteriography or guide catheter placement prior to attempted placement of a filter, and hence the results here reflect the risk of the procedure from signature of the consent form until the 30 day follow-up. There were 29 elective carotid stents performed outside of clinical trials during the study period. Twenty-eight of the patients were symptomatic with $>70 \%$ stenosis. They were not enrolled in trials for various reasons, ranging from bilateral stents placed in a single procedure in a patient with bilateral recent strokes, use of off-label devices (balloon expandable stents for ostial lesions), timing of the intervention and availability of the research staff (weekends), or patient preference. All of these patients had 30 day follow-up by the author, most with independent evaluation as part of the American College of Cardiology sponsored carotid artery revascularization (CARE) registry, and there were no 30 day strokes. They are not included because the follow-up was not standardized to the extent of the patients enrolled in the clinical trials.

Informed consent was obtained for all procedures. The research protocols were approved by the institutional review board.

Data were collected prospectively, with primary endpoints of stroke, death and MI in the first 30 days, as assessed independently by neurologists or research nurses certified in the National Institutes of Health Stroke Scale (NIHSS) and the Modified Rankin Scale. Follow-up at $24 \mathrm{~h}$ and 30 days was 100\%. All patients were seen by the treating neurointerventionalist at 30 days also. Additional events collected were access complications requiring surgery, transfusion or extended hospitalization, day of discharge for elective admissions and readmissions in the first 30 days. All patients had carotid ultrasound at 30 days also.

Preoperative imaging with CTA or MRA was routinely performed to assess the suitability of the aortic arch and carotid arteries for the procedure, and to plan the procedure. Patients in whom the lesion was excessively calcified, or in whom tortuosity of the aortic arch or internal carotid artery would prevent placement of a protection device, were not offered stent placement. All patients were pretreated with clopidogrel for 5-7 days and aspirin $325 \mathrm{mg}$ for at least 2 days, or alternatively 450 $600 \mathrm{mg}$ clopidogrel and $325 \mathrm{mg}$ aspirin at least $4 \mathrm{~h}$ prior to the procedure. Patients intolerant to clopidogrel received ticlopidine $250 \mathrm{mg}$ twice daily for 5 days. Patients were maintained on clopidogrel or ticlopidine for at least a month post procedure, and aspirin 325 or $81 \mathrm{mg}$ daily. Antihypertensive medications were usually held on the day of the procedure.

A variety of stents and embolic protection devices were used (table 1). All procedures were performed with a protection device in place.

Femoral access was used for all cases. Patients were given intravenous heparin until the activated clotting time was documented over $250 \mathrm{~s}$. A 6 F Cook Shuttle sheath was used in all cases. All lesions were pre-dilated with a 3-4 $\mathrm{mm}$ balloon followed by post-dilatation in most cases with a $4.5-5.0 \mathrm{~mm}$ balloon. In 26 cases no post-dilation was performed (10\%). No residual stenosis was the goal, but a residual stenosis of $30 \%$ or less was considered acceptable. 
Table 1 Stents and embolic protection devices used

\begin{tabular}{lc}
\hline Device & No of patients \\
\hline Acculink/Accunet & 116 \\
XACT/Emboshield & 30 \\
Precise/Angioguard & 78 \\
Precise/Proguard & 2 \\
\hline
\end{tabular}

All patients were admitted to a monitored bed for overnight observation.

\section{RESULTS}

Patient demographics and 'high risk' CEA criteria are summarized in tables 2 and 3 . Technical failure occurred in $1 / 227$ patients (0.4\%).

Four strokes occurred within 30 days of the procedure. The first occurred in a previously asymptomatic patient the morning after the procedure due to stent thrombosis and embolization to the left middle cerebral artery. This was treated with intra-arterial thrombolysis. The patient had a right parietal stroke. He was discharge to rehabilitation at 12 days. The NIHSS was 8 at $24 \mathrm{~h}$ and 2 at 30 days, but the patient was disabled due to neglect. The second occurred during the procedure for a symptomatic stenosis, with the onset of the neurologic deficit a few minutes after filter removal. MRI demonstrated multiple small embolic infarcts. She was discharged to rehabilitation at 7 days. The NIHSS was 0 at baseline, 9 at $24 \mathrm{~h}$ and 0 at 30 days after rehabilitation. The third patient presented and was hospitalized with vague symptoms of confusion. His imaging demonstrated an acute right vertebral dissection and subacute left posterior cerebral artery territory infarct in the left occipital lobe. He was found to also have a left carotid occlusion, severe right carotid stenosis and he subsequently developed a right hemispheric transient ischemic attack. The decision was made to treat the right carotid stenosis with CAS and the vertebral dissection with aspirin and clopidogrel. At $24 \mathrm{~h}$ after the CAS procedure, the patient extended the left posterior cerebral infarct into the left temporal lobe. He was discharged to home at 7 days. NIHSS prior to the CAS procedure was 2 (homonymous hemianopsia), 7 at $24 \mathrm{~h}$ and 2 at 30 days. There were no symptoms or MRI evidence of stroke in the right carotid territory. The fourth event occurred at 7 days and presented as dizziness, chest pain and stumbling speech. The diagnosis by the neurologist was a small cerebellar stroke. Symptoms resolved by discharge 3 days later. The patient had a dilated cardiomyopathy and was on Coumadin for that diagnosis and atrial fibrillation. The international normalised ratio was 1.7. MRI could not be obtained due to an implantable defibrillator. CT showed no acute change. He was admitted again with similar transient symptoms 1 week later, again with a subtherapeutic international normalised ratio. His neurologic

Table 2 Patient demographics

\begin{tabular}{lc}
\hline $\mathrm{n}$ & 226 \\
Men & $133(59)$ \\
Symptomatic & $82(36)$ \\
Asymptomatic & $144(64)$ \\
'Low risk' trials & $43(19)$ \\
Cardiopulmonary 'high risk' & $58(26)$ \\
Anatomic 'high risk' & $64(28)$ \\
Age (years) (range) & $41-89$ \\
Age $>80$ years & $29(13)$ \\
Age $>75$ years & $63(28)$ \\
\hline
\end{tabular}

Values are number (\%) unless otherwise stated.
Table 3 High risk criteria

\begin{tabular}{lc}
\hline Criterion & No (\%) of patients \\
\hline Severe emphysema & $28(12)$ \\
CHF class III/IV or EF $<30 \%$ & $15(7)$ \\
Coronary high risk (MI $<6$ weeks, & $15(7)$ \\
unstable angina, known 3 vessel CAD) & \\
Contralateral occlusion & $15(7)$ \\
Radiation & $9(4)$ \\
Neck dissection/tracheostomy & $12(5)$ \\
High or low (intrathoracic) lesions & $15(7)$ \\
Cervical immobility & $5(2)$ \\
Prior CEA & $20(9)$ \\
Severe bilateral carotid stenosis & $29(13)$ \\
Age $>75$ years & $63(28)$ \\
Age $>80$ years & $29(13)$ \\
\hline
\end{tabular}

CAD, coronary artery disease; $C E A$, carotid endarterectomy; $C H F$, congestive heart failure; $\mathrm{EF}$, ejection fraction; Ml, myocardial infarction.

examination was normal by a neurologist at the 30 day followup.

One patient had a sudden transient monocular visual disturbance 1 week after the procedure. Evaluation by an optometrist showed no evidence of retinal emboli and no discrete visual field abnormality. One patient with diabetes had a visual disturbance thought to be due to a small retinal hemorrhage in the eye ipsilateral to the carotid stent at the 30 day follow-up.

Cardiac events were as follows: one patient had a non- $Q$ wave MI 28 days following the CAS procedure. One patient was admitted to another hospital at 2 weeks for a congestive heart failure exacerbation. One patient was evaluated for chest pain in the emergency room at 2 weeks and discharged (EKG and enzymes negative). One patient had new onset of atrial fibrillation, asymptomatic, discovered on EKG required at 30 days.

Stroke, death and MI events are summarized in table 4.

One patient was readmitted with pneumonia within the first 30 days. There were two readmissions for gastrointestinal bleeding during the first 30 days. One had a gastrointestinal illness also and presented 3 weeks after the CAS procedure with dehydration, acute renal failure, anemia and altered mental status. She was treated with transfusion, hydration and intravenous antibiotics. She was discharged after 5 days with her renal function and neurologic status at baseline. One patient was admitted at 2 weeks with rectal bleeding requiring transfusion. Colonoscopy was negative. Aspirin was stopped on admission and no further treatment was required. A third patient with known chronic anemia received an outpatient transfusion during the first 30 days. One patient was admitted with an upper gastrointestinal bleed 45 days after the CAS procedure. There were no access complications requiring transfusion or surgery. The median fluoroscopy time for the CAS procedures was 9 min.

There were 206 patients admitted electively for the CAS procedure (the other 20 were already hospitalized from the index stroke). Two of the electively admitted patients had a stroke (already described above) and were discharged at days 12 and 7 . Of the other 206 patients, one was discharged after 2 days and one after 3 days, both due to prolonged hypotension. One patient was discharged after 2 days which was planned at the request of the family. One patient with known chronic anemia was found to be profoundly anemic on admission. The stent procedure was delayed for endoscopy which revealed sprue and transfusion. She was discharged on the third hospital day, the day after the CAS procedure. One patient was delayed for 1 day due to pharmacologically induced altered level of consciousness, which cleared 
Table 430 day stroke/death/myocardial infarction

\begin{tabular}{|c|c|c|c|c|c|c|c|}
\hline Patient & Symptomatic status & Procedure & Event & NIHSS baseline & MRS baseline & NIHSS 30 days & MRS 30 days \\
\hline 1 & Asymptomatic & Rt CAS & Ischemic stroke, Rt hemispheric & 0 & 0 & 2 & 3 \\
\hline 2 & Symptomatic & Rt CAS & Ischemic stroke, Rt hemispheric & 0 & 0 & 0 & 1 \\
\hline 3 & Symptomatic & Rt CAS & Ischemic stroke, Lt posterior temporal & 2 & 1 & 2 & 2 \\
\hline 4 & Asymptomatic & Lt CAS & Ischemic stroke, cerebellar & 0 & 0 & 0 & 0 \\
\hline 5 & Asymptomatic & Lt CAS & Non-0 Ml & 0 & 0 & 0 & 0 \\
\hline
\end{tabular}

CAS, carotid artery stenting; Lt, left; MI, myocardial infarction; MRS, Modified Rankin Scale; NIHSS, National Institutes of Health Stroke Scale; Rt, right.

after cessation of narcotics and anxiolytics given for back discomfort. The remainder of the patients were discharged on the first postoperative day. Hence 199/206 (97\%) patients electively admitted were discharged on the first postoperative day.

Of the 29 patients 80 years or older, 13 (45\%) were symptomatic. There was one stroke in this group, for an incidence of $3.4 \%$.

Overall, the 30 day incidence of stroke, death or MI for the entire group was $5 / 226(2.2 \%)$. The incidence of 30 day stroke in the symptomatic patients was $2 / 82(2.4 \%)$ and in the asymptomatic patients $2 / 144$ (1.4\%). Only two of the four strokes were in the territory of the revascularized carotid artery. The incidence of disabling stroke, defined as a Modified Rankin Scale $\geq 3$ at 30 days, was $1 / 226(0.4 \%)$. There were no deaths. The 30 day stroke and death rates compared with previously peer reviewed published data are presented in table 5.

\section{DISCUSSION}

The patient group reported here is one that would normally be considered relatively 'high risk' for carotid stenting. Twenty-nine of the patients in this series were over 80 years of age, which was associated with a $12 \% 30$ day stroke and death rate in the CREST lead-in phase. ${ }^{18}$ Thirty-six per cent were symptomatic. Cardiopulmonary 'high risk' criteria were present in 26\%. Given the 30 day major adverse event rates reported in published clinical trials previously, the combined 30 day stroke, death and MI rate of $2.2 \%$ reported here in clinical trial patients is lower than one might expect. The 30 day incidence of stroke and death in symptomatic patients was $2.5 \%$ and in asymptomatic patients $1.4 \%$, which compares favorably with CEA data from randomized trials. ${ }^{1-4} 1617$

Table 530 day rates of stroke and death of carotid stenting (myocardial infarction excluded)

\begin{tabular}{lcl}
\hline Study & $\begin{array}{l}\text { Symptomatic } \\
\text { patients (\%) }\end{array}$ & $\begin{array}{l}\text { Asymptomatic } \\
\text { patients (\%) }\end{array}$ \\
\hline ARCHeR & 11.6 & 5.4 \\
CAPTURE & $12^{*}$ & $5.4^{*}$ \\
BEACH & 7.5 & 5 \\
CREATE & 15 & 3.4 \\
CREST lead-in & 5.7 & 3.8 \\
SPACE & 6.8 & $\mathrm{~N} / \mathrm{A}$ \\
EVA-3S & 9.6 & $\mathrm{~N} / \mathrm{A}$ \\
Current report & 2.5 & 1.4 \\
\hline
\end{tabular}

*Includes myocardial infarction. Stroke and death were not reported separately for asymptomatic and symptomatic patients. The incidence of myocardial infarction in CAPTURE was $0.9 \%$ for all patients.

ARCHeR, Acculink for Revascularization of Carotids in High Risk Patients; BEACH, Boston Scientific EPI: A Carotid Stenting Trial for High Risk Surgical Patients; CAPTURE, Carotid Acculink/Accunet Post-Approval Trial to Uncover Unanticipated or Rare Events; CREATE, Carotid Revascularization with EV3 Technology Evolution; CREST, Carotid Revascularization Endarterectomy versus Stenting Trial; SPACE, Stent-Supported Percutaneous Angioplasty of the Carotid Artery versus Endarterectomy.
There are several possible reasons for the discrepancy between the adverse event rates reported here and the higher rates in other trials. Experience in cerebrovascular interventions may play a role. The credentialing criteria for CREST have been published and are the most rigorous of the CAS trials. ${ }^{20-21}$ This is likely why results in the CREST lead-in phase are superior to the published post-market and pre-market approval data. However, the lead-in data by definition involve operators gaining experience in CAS procedures or with the specific device, as the purpose of the lead-in phase was to eliminate the learning curve as a possible cause of additional risk in the CAS group. In the EVA-3S trial, an interventionalist qualified with a career experience of: (1) 12 carotid stents; (2) five carotid stents and 35 interventions elsewhere; (3) $<5$ carotid stents but have a tutor present. Incredibly, a physician who had never done a cerebral arteriogram or a carotid stent could do one in the EVA-3S trial. The CAS procedure in EVA-3S was a technical failure in 13/261 cases $(5 \%)$, testifying to the inexperience of the interventionalists. Interventionalists in the SPACE trial qualified with 25 percutaneous interventions but it is unclear if these had to be carotid stents. Physicians in CAPTURE had varying levels of experience. Operators were separated into level 1,2 or 3 based on experience. Level 1 operators participated in previous clinical trials, level 2 operators had performed at least 10 CAS procedures and level 3 operators had performed 25 carotid arteriograms and had experience with self-expanding stents and 0.014" (wire systems). The level 3 investigators are by definition inexperienced in carotid stenting, and one could argue that the level 2 definition permits inexperienced operators as well, as no requirement for experience with cerebral angiography is included. While the authors of CAPTURE concluded that there was no difference in outcomes between the three groups, the trend in every important outcome measure except MI was towards higher complications in the lesser experienced operators. The incidence of stroke, major stroke and death increased inversely to experience level. It bears repeating that the 30 day death rate was $2.3 \%$ in the least experienced group.

The interventionalist in the series reported here had performed hundreds of cerebral arteriograms and had extensive experience with guide catheter placement in the cerebral vessels, and had performed 86 carotid stent procedures at the institution prior to enrolling the first patient in a trial.

Use of protection devices was not mandated in EVA-3S or SPACE, and this may have led to higher complication rates. Although the SPACE investigators did not report a higher incidence in the group without protection, protection devices were used in a minority of patients. Protection devices were mandated in the CREST lead-in phase and in all of the pre-market approval or postmarket surveillance trials. Use of protection devices can definitely prevent strokes, but they do add complexity to the procedure which may negate their benefit for inexperienced operators.

A conservative approach to stent post-dilatation was employed in this series. A balloon of $4.5-5 \mathrm{~mm}$ was used in $99 \%$ 
of cases, and in $10 \%$ of cases there was no post-dilatation. This strategy may have contributed to the low complication rate, but has not been associated with higher rates of restenosis. ${ }^{5}$

Non-invasive preoperative imaging of the aortic arch and carotid arteries may also have contributed to a low complication rate. While only a small percentage of patients are excluded from consideration for CAS after non-invasive imaging, these patients are also likely to be those that would be at higher risk from a CAS procedure. This strategy also allows planning of the entire intervention prior to puncture, and avoids unnecessary catheterization of non-target vessels, allowing a shorter and potentially safer procedure.

In summary, series such as the one reported here indicate that carotid stenting can be very safe in the community. The strategy employed here, including preoperative non-invasive imaging of the aortic arch and carotid arteries, use of cerebral protection devices and a conservative approach to post-dilatation, is recommended. The level of experience in cerebral angiography and carotid stenting required to produce results comparable to CEA may be higher than previously thought.

Competing interests None.

Ethics approval This study was conducted with the approval of the Institutional Review Board, Forsyth Medical Center.

Provenance and peer review Not commissioned; not externally peer reviewed.

\section{REFERENCES}

1. North American Symptomatic Carotid Endarterectomy Trial Collaborators. Beneficial effect of carotid endarterectomy in symptomatic patients with high-grade carotid stenosis. N Engl J Med 1991;325:445-53.

2. European Carotid Surgery Trialists' Group. Interim results for symptomatic patients with severe (70-99\%) or with mild (0-29\%) carotid stenosis.

3. Executive Committee for the Asymptomatic Carotid Atherosclerosis Study. Endarterectomy for asymptomatic carotid artery stenosis. JAMA 1995;273:1421-8.

4. MRC Asymptomatic Carotid Surgery Trial (ACST) Collaborative Group. Prevention of disabling and fatal strokes by successful carotid endarterectomy in patients without recent neurological symptoms: a randomized controlled trial. Lancet 2004;363:1491-502.

5. Heck D. Incidence and time course of carotid in-stent restenosis in a consecutive series of 295 patients. J Neurointervent Surg 2009;1:44-7.

6. De Donato G, Deloose J, Setacci F, et al. Long term results of carotid artery stenting J Vasc Surg 2008:48:1431-40.

7. Setacci C, Chisci E, Setacci F, et al. Grading carotid in-stent restenosis: a 6 year follow-up study. Stroke 2008;39:1189-96.

8. Hobson RW, Brott TG, Roubin GS, et al. Closure of the Lead-in phase of CREST: 30 day and 1 year analyses. Stroke 2008:39:557.

9. Ringleb PA, Hennerici M, Stingele R, et al. Stent-protected percutaneous angioplasty verses endarterectomy of symptomatic carotid artery stenosis: two year results of the SPACE trial with respect to subgroup analysis. Stroke 2009;40:e108.

10. Gurm HS, Yadav JS, Fayad P, et al. Long term results of carotid stenting versus carotid endarterectomy in high risk patients. N Engl J Med 2008;358:1572-9.

11. Yadav JS, Wholey MH, Kuntz RE, et al. Protected carotid-artery stenting versus endarterectomy in high-risk patients. N Engl J Med 2004;351:1493-501.

12. White CJ, lyer SS, Hopkins LN, et al. Carotid stenting with distal protection in high surgical risk patients: The BEACH trial 30 day results. Catheter Cardiovasc Interv 2006;67:503-12.

13. Gray WA, Hopkins LN, Yadav JS, et al. Protected carotid stenting in high-surgical-risk patients: The ARCHeR results. J Vasc Surg 2006;44:258-68.

14. Gray WA, Yadav JS, Verta P, et al. The CAPTURE Registry: results of carotid stenting with embolic protection in the post approval setting. Catheter Cardiovasc Interv 2007;69:341-8.

15. Safian RD, Bacharach JM, Ansel GM, et al. Carotid stenting with a new system for stenting and distal embolic protection in high risk patients: the carotid revascularization with ev3 technology evolution (CREATE) feasibility trial. Catheter Cardiovasc Interv 2004;63:1-6.

16. SPACE Collaborative Group. 30 day results from the SPACE trial of stent-protected angioplasty versus carotid endarterectomy in symptomatic patients: a randomized non-inferiority trial. Lancet 2006:368:1239-47.

17. Mas JL, Chatellier G, Beyssen B, et al. Endarterectomy versus stenting in patients with symptomatic severe carotid stenosis. N Engl J Med 2006;355:1660-71.

18. Howard VJ, Voeks JS, Lutsep HL, et al. Does sex matter? 30 day stroke and death rates after carotid stenting in women versus men. Stroke 2009;40:1140-7.

19. Hobson RW, Howard VJ, Roubin GS, et al. Carotid artery stenting is associated with increased complications in octogenarians: 30 day stroke and death rates in the CREST lead-in phase. J Vasc Surg 2004:40:1106-111.

20. Al Mubarak N, Roubin GS, Hobson RW, et al. Credentialing of carotid stent operators for the carotid revascularization endarterectomy verses stenting trial (CREST). Stroke 2000; 31:292

21. Roubin GS, Ferguson RD, Chakhtoura E, et al. Credentialling and training of the interventionalists in the CREST trial. Stroke 2009;40:e108. 